\title{
LA MEMORIA EDUCATIVA Y PEDAGÓGICA COMO MODELO DIDÁCTICO PARA LA FORMACIÓN INICIAL DEL PROFESORADO
}

THE EDUCATIONAL MEMORY AS A DIDACTIC MODEL FOR TEACHERS' INITIAL TRAINING

\author{
María Teresa Bejarano Franco \\ UNIVERSIDAD DE CASTILLA-LA MANCHA \\ MariaTeresa.Bejarano@uclm.es \\ JAVIER RODRÍGUEZ TORRES \\ UNIVERSIDAD DE CASTILLA-LA MANCHA \\ Javier.RTorres@uclm.es
}

\section{RESUMEN}

Desde este artículo, se plantea la importancia de la utilización de la memoria educativa y pedagógica como modelo didáctico en los estudios de Grado de Maestros (Educación Infantil y Primaria). El trabajo didáctico a partir de este modelo invita al alumnado a la adquisición de competencias relacionadas con la investigación, tarea relevante en su formación inicial. Este modelo, les pone en relación con fuentes primarias de conocimiento y les permite la indagación escolar. Se trata de justificar la "presencia" del modelo en determinadas materias pedagógicas para recuperar huellas didácticas institucionales y profesionales a través de la memoria escolar partiendo de las fuentes orales y del análisis documental. Así se facilita la reflexión sobre el pasado reciente educativo y se recuperan aportaciones para la escuela actual.

En este trabajo señalamos la huella histórica de la Institución Libre de Enseñanza mediante el testimonio de Luis de Azcárate, alumno de la última promoción de la Institución. Su discurso constituye un auténtico legado personal y pedagógico que hace resignificar una parte de la historia educativa que, en muchas ocasiones, permanece guardada y silenciada.

\section{PALABRAS CLAVE}

Competencias, formación del profesorado, historia de la educación, investigación educativa, memoria escolar 


\begin{abstract}
As result of our involvement in an international project, the influence of educational memory is considered as a didactic model for teacher degree (pre-school education and primary school). Didactic model encourages the students in research work as an important task in their early education.

Didactic model plays a significant role in the contacts between the students and the early knowledge and it allows to scholar research.

Professional and institutional teaching footprints will be recovered trying to justify didactic model. It will be made through school age memory, which is based in oral sources and documental analysis.

This way, the consideration about educational recent past is facilitated and contribution of knowledge is recovered for present-day school.

By Luis de Azcarate testimony, last promotion student of the institution, historic footprint of Institución Libre de Enseñanza is analysed in this essay.

Luis de Azcarate dissertation's is a right personal and educational legacy, which emphasises a part of educative history, but in many cases, we have a feeling it must be academically kept and silent for any interest.
\end{abstract}

\title{
KEY WORDS
}

Competences, teacher training, education history, educational research, school memory

\section{INTRODUCCIÓN}

Se presenta un artículo que parte de la producción de datos obtenidos en el marco de un proyecto de investigación internacional ${ }^{1}$. El principal objetivo de este texto es plantear el beneficio pedagógico que implica introducir el modelo centrado en la memoria educativa y pedagógica como medio didáctico en el Grado de Maestros (Infantil y Primaria), concretamente en las asignaturas implicadas en las áreas de Didáctica y Teoría e Historia de la Educación.

1 El artículo es fruto de un proyecto internacional denominado: Reposición de trazas e itinerarios didácticos en la formación universitaria (UBA) para la enseñanza de la psicología. Interpelaciones al desdibujamiento de la Carrera de Profesorado (1957-1976). Proyecto de investigación financiado por UBACyT. Programación Científica 2014-2017. Resolución (CS) $\mathrm{N}^{\circ} 1271 / 14$. 
Se aproximan las bases teóricas de este modelo, y se profundiza en la descripción de dos de sus principales técnicas: la documental y los testimonios orales. El artículo muestra las ventajas del uso didáctico de ambas técnicas para la recuperación de hallazgos e hitos de instituciones que aportaron valía a la educación española, es el caso de la Institución Libre de Enseñanza. Por último, se acude a la palabra como un instrumento cargado de conocimiento a partir de la voz y la memoria educativa de un alumno de la ILE.

\section{LA MEMORIA EDUCATIVA Y PEDAGÓGICA COMO MODELO DIDÁCTICO PARA LA ENSEÑANZA UNIVERSITARIA}

El EEES "ha comprometido" los procesos didácticos en las aulas universitarias. Ello significa, entre otras cosas, que ha hecho que los docentes tengamos que optar por modelos más activos para la enseñanza. La revisión bibliográfica sobre metodologías basadas y-o adaptadas al EEES, nos aproxima a un novedoso mapa de herramientas metodológicas al servicio de la transmisión del conocimiento en los Estudios de Grado. Sabemos que los modelos tradicionales basados en la magistralidad están dando paso a otros como: el ABP (Bejarano \& Lirio, 2008), proyectos de trabajo como herramientas de evaluación y aprendizaje (Rodríguez, 2014), los seminarios y talleres (Alfaro, 2009), la metodología colaborativa (Portal, 2011), o el aprendizaje en red también conocido como e-learning y aprendizaje virtual (Cabero \& Gisbert, 2005). Cada modelo ha venido desarrollando sus propias técnicas y estrategias con el objetivo de dinamizar el paradigma de enseñanza / aprendizaje basado en la adquisición de competencias (Bolívar, 2008; Riesco, 2008). Ello ha implicado dar mayor protagonismo al alumnado universitario perfeccionando habilidades de pensamiento crítico, reflexivo, imaginativo y sensitivo (Gómez et al, 2004). Desde estos planteamientos se pide a los discentes, más implicación en su propio proceso formativo. Pero quizá, esté llegando la hora de preguntarnos si el aprendizaje adquirido mediante estos "formatos metodológicos" está llevando al alumnado a interaccionar y experimentar con las fuentes primarias de producción de conocimiento que le proporcionan un contacto directo con los procesos de interpretación e indagación escolar. Esta pregunta se hace especialmente relevante cuando está enfocada para la formación inicial del profesorado (Grado de Magisterio Infantil y Primaria) ya que en estos Estudios, se destacan las siguientes competencias $^{2}$ para que sean alcanzadas por el alumnado:

${ }^{2}$ Las competencias y el objetivo de aprendizaje, se extraen de las Memorias de Verificación del Grado de Infantil y Primaria. Recuperadas de: 
- 1.2.1.1. II. 4. Adquirir conocimientos sobre la evolución del pensamiento, las costumbres, las creencias y los movimientos sociales y políticos a lo largo de la historia.

- II.4. Conocer la evolución histórica del sistema educativo en nuestro país y los condicionantes políticos y legislativos de la actividad educativa.

Así como el siguiente objetivo de aprendizaje:

- Relacionar la evolución histórica de la educación en España con el contexto socio-educativo.

Las competencias mencionadas y el resultado de aprendizaje señalado, se ubican en la parcela dedicada a la formación pedagógica, y ello conlleva la interpretación de los contextos escolares desde los hechos significativos socioeducativos. Para ello, se podría incorporar el modelo de enseñanza basado en la memoria educativa y pedagógica de la escuela. Se recogen ya en nuestro contexto, publicaciones educativas que señalan los beneficios de aplicación didáctica mediante esta metodología (Sánchez Morillas, 2010; Sánchez de Madariaga, 2012). Lo que significa propiciar en el alumnado el aprendizaje sobre prácticas, experiencias y dinámicas educativas que acontecieron en un tiempo pasado y que perviven y dan sentido a distintas tipologías escolares en la actualidad.

Este modelo cuenta, desde hace algún tiempo, con técnicas para la extracción y producción de datos, tales como la documentación narrativa de prácticas escolares (Dávila, 2011; Grangeiro, 2011; Suárez, 2011) y la entrevista narrativa-biográfica (Pujadas, 2000; Bustos, 2010; Gómez, 2012) que poseen una doble dimensión: la de la enseñanza y la de la investigación. He ahí también la doble importancia de trabajar con ellas en el Grado de Infantil y Primaria, por cuanto hay que enfatizar que la formación inicial de maestrosas debe "ser nutrida" por una sólida formación en investigación. La investigación se viene considerando como uno de los contenidos más relevantes en la formación inicial del profesorado (Stenhouse, 1987; Latorre \& González, 1992). Incorporar la investigación a la formación inicial de maestros y maestras permite obtener conocimientos sobre la complejidad educativa (Bejarano, Mateos \& Rodrígues, 2015). Existe una constante preocupación institucional y social por mejorar los resultados académicos en los niveles de

http://www.uclm.es/cr/educacion/gradoEducacionInfantil.asp http://www.uclm.es/cr/educacion/gradoEducacionPrimaria.asp 
educación obligatorios y por reducir las tasas de abandono escolar (Fernández Enguita, Mena \& Riviere, 2010). Para alcanzar estos objetivos, parece imprescindible que la educación y la investigación sean la cara de una misma moneda.

Aludimos a la documentación narrativa de prácticas escolares y a la entrevista narrativa-biográfica, también denominada historia oral, como técnicas dentro de este modelo (memoria educativa y pedagógica de la escuela) ya que proporcionan la producción y aproximación a datos experienciales y reales. La aplicación de estas técnicas para la enseñanza en las materias de Pedagogía en el Grado de Maestro (Educación Infantil y Primaria) habilita un espacio de conversación e interpelación que permite poner en relación al alumnado con experiencias e instituciones educativas muy significativas en el pasado y que han ido dejando huellas didácticas poco visibles en la actualidad. Es el caso de la Institución Libre de Enseñanza, cuyas aportaciones son poco recogidas en los textos de Educación en el área de la Historia Educativa.

Los aportes teóricos y metodológicos del modelo para la enseñanza y la investigación presentados en este artículo, posibilitan la recuperación de las huellas didácticas en forma de documentos curriculares, administrativos y personales; y el acceso a informantes que habitaron estas experiencias educativas. Se trata de reconstruir la memoria de la escuela (Aguirre, 2013). Un buen instrumento para esa reconstrucción es la perspectiva biográfica o también llamada historia oral (Diamant, 2010). Las fuentes orales vienen permitiendo que los estudiantes puedan tener acceso de un modo más cercano a los cambios operados en la vida cotidiana escolar y facilita que se inicien en la reflexión sobre aspectos del pasado.

\section{3. ¿POR QUÉ TRABAJAR CON LA HISTORIA ORAL EN LA FORMACIÓN INICIAL DE MAESTROS Y MAESTRAS?}

Trabajar con las diversas formas de historia oral educativa y las fuentes documentales que las acompañan en forma de documentos personales e institucionales; supone tener acceso a una parte de la memoria colectiva con la que poco o nada se trabaja en las aulas universitarias. Aguilar nos orienta sobre el poder que tiene la memoria colectiva como recurso de acceso al conocimiento: 
La memoria colectiva es el proceso social de reconstrucción del pasado vivido y experimentado por un determinado grupo, comunidad o sociedad (...) la memoria colectiva insiste en asegurar la permanencia del tiempo y la homogeneidad de vida (...) la memoria es comunicativa (...) los grupos tienen la necesidad de reconstruir permanentemente sus recuerdos a través de sus conversaciones, contactos, rememoraciones. (...) Toda memoria e incluso la individual se gesta y se apoya en el

Estos procesos de recuperación de hechos y datos, nos aproximan a los conocimientos que están guardados en el olvido y nos indica cuál es el punto de partida educativo y contextual de los hoy llamados modelos innovadores para la enseñanza y que se "localizan", entre otros en: las Comunidades de Aprendizaje, Waldorf o las Cooperativas escolares. Sabemos de los orígenes de estas experiencias educativas porque hemos mirado al pasado desde las fuentes secundarias de información y la mirada nos ha devuelto el origen de estas experiencias en los principios de las escuelas democráticas y en la Escuela Nueva. Ello ha sido posible por el rastreo de signos y huellas educativas recuperadas a través de la investigación historiográfica (Bolívar \& Domingo, 2006).

Pero, ¿se utilizan las fuentes orales como recurso para la enseñanza de aula? Nuestro contexto educativo universitario no ha introducido los testimonios como instrumentos didácticos. Algo que si es más frecuentes en otros contextos como los latinomericanos (Benadiba \& Plotinsky, 2001; Walter, 2002; Meyer, 1991, 1995)

Sin embargo, las instituciones escolares, son una fuente valiosa de recursos para la historia oral, dado que cuentan con innumerables actores y vivencias que pueden ofrecer testimonios y convertirse en instrumentos de producción de datos que ayudan a comprender las realidades y hechos históricos. La historia oral reformula el presente y ayuda a dar sentido y continuidad a lo que hoy ya existe en la escuela. Además da viveza al conocimiento teórico. Ana Diamant, nos recuerda que:

En momentos de acelerados cambios y en los que coexisten diversas temporalidades, variadas formas de relato y múltiples posibilidades de registro, se intentará la salvaguarda de información a partir de la transmisión oral de hechos sucedidos en la escuela y en su entorno, desde narraciones testimoniales de recuerdos de historias aprendidas, transmitidas y vividas en diferentes espacios y tiempos, procurando esclarecer tramos del pasado reciente para que a la par de ser 
preservados puedan ser utilizados en nuevas situaciones escolares para explicar el presente. $(2014,7)$

La historia oral en el aula y como recurso didáctico, nos hace retomar el mundo simbólico anclado en el pasado que da cuenta de las realidades presentes, se recuperan las experienciales y las acciones educativas que fueron posibles décadas atrás; se pone nombre a los y las protagonistas que transitaron por la historia educativa, se rescata el olvido significativo, se recupera parte del presente, se trabaja con personas mayores que ponen su herencia en forma de recuerdo y palabra al servicio del conocimiento para las generaciones más jóvenes.

\section{RESCATANDO A LA INSTITUCIÓN LIBRE DE ENSEÑANZA A TRAVÉS DE LA MEMORIA HISTÓRICA EDUCATIVA DE NUESTRO PAÍS}

Desde nuestro trabajo referido a la memoria escolar a través del proyecto de investigación, del cual parte este artículo, hemos rescatado instituciones educativas con el objetivo de actualizar principios pedagógicos y prácticas didácticas que han tomado significado en las leyes educativas progresistas de nuestro contexto. Intentando comprender la realidad educativa actual hemos puesto la mirada historiográfica en una época pasada y hemos detectado continuidad en algunos modelos escolares de la actualidad. Ello se ha venido reforzando con apasionantes testimonios recogidos por el alumnado que estuvo presente en los modelos educativos de los que nos hemos hecho eco. Así, para recuperar la Institución Libre de Enseñanza nacida en 1879 (en adelante ILE) también hemos aplicado la entrevista a modo de historia de vida, como fuente de recogida de datos. Ofrecemos el ilustrativo testimonio de uno de sus alumnos, Luis de Azcárate ${ }^{3}$. El acceso a su testimonio nos posibilitó la reposición de trazas e itinerarios didácticos en la ILE.

Me eduqué en la Institución Libre de Enseñanza, termino en la Institución Libre de Enseñanza en julio. Soy la última generación que salió de la séptima clase... Entonces, empieza la guerra, a mí me coge la guerra en Ginebra, veraneando con Fernando de los Ríos que quería ir a descansar a la casa de mi tío Pablo, que era Secretario General del adjunto de la Sociedad de Naciones". (Entrevista, diciembre de 2014)

\footnotetext{
${ }^{3}$ Ofrecemos las palabras de Luis de Azcárate por entender que es el ejemplo más próximo a lo que queremos significar en este artículo. La investigación, nos posibilitó acceder a otras alumnas como: Isabel Luzuriaga, Lucía PiosevPrevich, o Laura Cruzalegui, entre otras. La entrevista a este alumno tiene fecha de diciembre de 2014 y tuvo lugar en el domicilio del informante (Pozuelo de Alarcón, Madrid).
} 
Con su discurso, entregado en forma de legado personal y pedagógico, nos adentró a revivir con él su infancia y experiencias educativas calificadas como únicas y que se produjeron en los locales educativos de la ILE. Para entender este legado y su «efecto contagio», es preciso que desentrañemos los contextos, personajes y hechos que dieron cabida al origen y desarrollo de la ILE. Para ello, hemos recurrido a las fuentes documentales de primer orden. Aproximar la ILE a las aulas en la formación inicial de maestros y maestras desde las técnicas que nos ofrece el modelo de memoria escolar y pedagógica, tiene el valor de resignificar una parte de la historia educativa en nuestro contexto que parece estar guardada en el silencio académico.

Antes de profundizar en las señas de identidad educativas que este alumno, discípulo entre otros de Cossío, nos ha dejado desde la oralidad, queremos aproximarnos a las bases pedagógicas de la propia Institución. Comenzamos a trabajar sobre la concepción educativa y educadora que la propia ILE recoge en el artículo 15 de sus Estatutos (1876):

La Institución Libre de Enseñanza es completamente ajena a todo espíritu e interés de comunión religiosa, escuela filosófica o partido político; proclamando tan sólo el principio de la libertad e inviolabilidad de la Ciencia y de la consiguiente independencia de su indagación y exposición respecto de cualquiera otra autoridad que la de la propia conciencia del profesor, único responsable de sus doctrinas.

Para ejercitar esta hoja de ruta educativa, fue preciso dar forma y diseñar todo un programa educativo que, a su vez, rompía con antiguos esquemas didácticos y abría horizontes a una sociedad necesitada de una ciudadanía bien formada, alfabetizada y capaz de liderar procesos de cambios complejos $\mathrm{y}$, en muchas ocasiones, convulsos que acaecerán al final del siglo XIX y comienzos del XX.

Los protagonistas en hacer realidad los cambios, su persistencia e influencia en posteriores generaciones tienen importancia por méritos propios y una significativa entidad en la historia de la educación española de los siglos XIX y XX. Son los indiscutibles actores y actrices del proceso de renovación histórica, social y educativa iniciada en España con la revolución de septiembre de 1868 (Jiménez, 1985) y que se desarrollará hasta llegar al primer tercio del siglo XX con el luctuoso episodio de la Guerra Civil (1936).

Se hace preciso recoger la realidad ILE a través del acercamiento de la memoria escolar a los actuales maestros y maestras en formación ello implica también, aproximar la memoria sociológica española. El contexto del 
nacimiento de la ILE, es característica de una sociedad agrícola, en la cual el poder político lo ostentaban las clases acomodadas y la Iglesia. Los liberales del s. XIX tienden al abandono educativo en el diseño por generar progreso en la nación. El precio a pagar fue muy caro: un país analfabeto, con tendencia al enfrentamiento fratricida y una Iglesia Católica vigilante de la ignorancia con afán inquisitorial que impedirá, por otra parte, la libertad de prensa e imprenta. Con este panorama la tan apreciada escuela pública, en aquel momento histórico queda reducida a casi nada.

Hablar desde las aulas universitarias sobre el pasado educativo, nos compromete también hablar de la Universidad de época que, reforzó los privilegios de unos pocos. Además, proponía la promoción directa y casi exclusiva a puestos de poder académicos, a quienes con fortuna podían formarse en ella. El profesorado universitario utilizó su cátedra como plataforma política, evadiendo cuestiones sociales y pedagógicas; como consecuencia la docencia fue totalmente rutinaria y el método más innovador se fundamentó en el protagonismo y fomento de la capacidad memorística.

Pero, los aires renovadores de Sanz del Río ${ }^{4}$ (1814-1869) favorecerán que algunos intelectuales de esta época convulsa, en el orden político y académico, hereden una filosofía sistémica, una moral laica y que la apliquen con el objetivo de desarrollar la conciencia individual y firmes convicciones sobre el papel que debe cumplir la educación en el país y que no era otra que la de solucionar gran parte de los males de España. (Jiménez, 1985).

Algunos de esos intelectuales como Francisco Giner de los Ríos (1839-1915), Gumersindo de Azcárate y Nicolás Salmerón, Catedráticos de Universidad y criticados por ejercer la libertad cátedra ${ }^{5}$ fueron separados de ella por negarse a ajustar sus enseñanzas a los dogmas oficiales en materia religiosa, política o moral. Ellos conformarán la nómina de los primeros institucionalistas consagrados al cultivo y propagación de: la Ciencia y la igualdad educativa, entre otros principios. Iniciarán su tarea educadora, fuera de la Universidad,

\footnotetext{
${ }^{4}$ Julián Sanz del Rio Catedrático de Filosofía, estudioso de las tendencias más innovadoras del momento, viaja por Europa y permanece una larga temporada en Heildelberg, sin duda el principal núcleo del krausismo en Alemania. A su vuelta tradujo algunas obras del mismo Krause y constituye con sus discípulos un círculo de marcado carácter krausista, entre los más destacados está Giner de los Ríos. El fundamento del krausismo es el racionalismo armónico, liberalismo político y económico, organismo social y revolucionismo y reformismo social.

${ }^{5}$ Decreto y Circular (fechadas el 26 de febrero de 1876) del Ministro Manuel de Orovio, por los que se limita la libertad de cátedra, producen las protestas de algunos Catedráticos y los expedientes contra ellos.
} 
creando un establecimiento de enseñanza y un movimiento de innovación pedagógica (Jiménez-Landi, 1973). Constituirán una escuela modelo, donde cursar desde la enseñanza de párvulos hasta estudios universitarios, sin que existiera discontinuidad, de acuerdo con los principios de unidad $^{6}$ escolar, de la Ciencia y evolutiva en el individuo. El método intuitivo, el principio de actividad, el valor del trabajo, la inserción en la realidad, la supresión de exámenes y la coeducación, las excursiones y visitas a museos, fueron las líneas básicas de la pedagogía institucionalista. De estos principios dará cuenta Azcárate en el siguiente epígrafe. Unos planteamientos muy innovadores que anteceden en la Escuela Nueva.

Desde el inicio de la ILE (1876) y con el impulso revolucionario en las conciencias, académicas como la de Francisco Giner de los Ríos (Cossío, 1966), se inicia una dinámica progresista imparable en el contexto educativo del país. Se crean distintos establecimientos educativos, formativos e investigadores, se generan intervenciones pedagógicas innovadoras que relatamos en orden cronológico en el esquema adjunto a este artículo.

El análisis documental nos ha permitido acceder a El Boletín de la Institución Libre de Enseñanza (BILE) y saber que este Boletín comenzó a publicarse cuatro meses después de la fundación de la ILE, el 7 de marzo de 1877. Entre sus objetivos más destacables está el de informar sobre la marcha de la propia Institución y responder a la necesidad de publicaciones científicas para dar a conocer el movimiento intelectual contemporáneo. Con este enfoque y premisas el Boletín, pronto se convirtió en una publicación muy importante en el mundo editorial español. Lo avaló el carácter cosmopolita y multidisciplinar que tenía. Además contaba con una gran calidad intelectual entre sus colaboradores de formación pedagógica, investigadora, filosófica y literaria. Entre otros colaboradores contó en sus páginas con las aportaciones de: Bertrand Russell, Henri Bergson, Charles Darwin, John Dewey, Santiago Ramón y Cajal, Miguel de Unamuno, María Montessori, León Tolstoi, H. G. Wells, Rabindranaz Tagore, Juan Ramón Jiménez, Gabriela Mistral, Benito Pérez Galdós, Emilia Pardo Bazán, Azorín, Eugenio D'Ors...

A partir de 1889, el contenido de este documento, quedó estructurado en tres secciones permanentes:

\footnotetext{
${ }^{6}$ En referencia al ámbito escolar será determinante la asunción y desarrollo de estas ideas por parte de Lorenzo Luzuriaga reflejadas claramente en su obra La Escuela Nueva Pública, publicada en 1931, mediante la concreción de la unificación de las diversas instituciones educativas, desde la escuela de párvulos a la universidad, estableciendo puntos de enlace entre ellas, aunque conservando cada una su fisonomía y particularidad propia.
} 
- Pedagogía, dedicada a temas de enseñanza

- Enciclopedia, que recogía lo relacionado con la ciencia, el arte, la filosofía, la historia, la arqueología y otras disciplinas.

- Institución, que trataba los temas referentes a la vida de la propia ILE.

En 1882 se abren las puertas del Museo Pedagógico, que aún forma parte de la memoria pedagógica de este país. Este Museo, se entrega a la tarea del cultivo de la Pedagogía y las Ciencias. También se utiliza como centro de investigación y enseñanza, contando con la enorme labor de su director Manuel Bartolomé Cossío (1957-1935). Entre sus experiencias educativas más destacadas y conocidas está la introducción de las colonias escolares a partir de 1887, la creación de un laboratorio de antropometría y psicología experimental, otro de física y química, de esto también hablará Azcárate desde su testimonio. Además de cursos de perfeccionamiento para maestros en ejercicio y para inspectores. Inaugurando un nuevo siglo, a instancias de la ILE y para dar más cientificidad a la Pedagogía, se crea con carácter oficial la Cátedra de Pedagogía Superior en 19017, dentro del Doctorado de la Facultad de Filosofía y Letras de Madrid, concibiéndose como un laboratorio pedagógico con carácter práctico. Se nombra Catedrático a Manuel Bartolomé Cossío, autorizando que se impartiera Pedagogía en el Museo Pedagógico, donde también se impartían enseñanzas de la Cátedra de Psicología Experimental.

Por otro lado, la necesidad de reformar la Universidad a través de su profesorado, hace que se cree la Junta de Ampliación de Estudios e Investigaciones Científicas en 1907 (Millán, 1983; Sánchez Sánchez, 2012). Fue impulsada por Giner de los Ríos y sus colaboradores. Ramón y Cajal fue nombrado presidente y a José Castillejo ${ }^{8}$ (1877-1945) secretario, convirtiéndose en el verdadero artífice de la Junta, además de ser un estrecho colaborador de Giner de los Ríos. Siguiendo a Palacios (1979, 30-31) queremos destacar la figura de Castillejo, Castillejo es la persona que Giner elige para llevar a cabo muchos de sus proyectos educacionales. Es, así, el hombre clave en la última etapa de la Institución. La Junta supone una oportunidad para

\footnotetext{
${ }^{7}$ Decreto de 30 de abril de 1901.

${ }^{8}$ Hacemos una breve reseña a este importante institucionalista debido a que es nacido en Ciudad Real (1877). Procede de una familia acomodada, su padre ejercía la abogacía y su madre estaba emparentada con la familia del poeta Ángel Crespo. Fue el mayor de cuatro hermanos, estudió bachillerato en Ciudad Real. Es señalado como uno de los más acérrimos militantes en la pedagogía de la ILE.
} 
modernizar la Universidad y construir una auténtica política de investigación (Otero, 2007). Se empezaron a mandar becarios postgraduados al extranjero (unos 2000 entre 1910-1936) y se crearon centros de investigación que hoy en día se catalogarían como la base del Consejo Superior de Investigaciones Científicas.

La Junta del Patronato en 1910, decide crear otro establecimiento, una Residencia de Estudiantes concebida como colegio universitario con un doble objetivo: posibilitar una formación más completa en los universitarios y generar un ambiente familiar facilitador del estudio (Jiménez, 1985). Al cargo de la dirección estuvo Alberto Jiménez Fraud (1833-1964), otro destacado pedagogo de la ILE. Por la sala de conferencias pasaron las más altas personalidades de la cultura española y extranjera, entre otros: Alberto Jiménez logró que Henri Bergson hablara a los residentes. Posteriormente pasaron por la Residencia, Einstein, Howard Carter, Gilbert Keith Chesterton, Paul Valéry, Marie Curie, Ígor Stravinski, Paul Claudel, Louis de Broglie, Herbert George Wells, Max Jacob, Le Corbusier, Keynes... Fueron residentes Alfonso Reyes Ochoa, Julián Besteiro, Santiago Ramón y Cajal, Manuel de Falla, Unamuno, Eugenio d'Ors, Federico de Onís, Valle-Inclán, Juan Ramón Jiménez, Manuel Machado, León Felipe, Zulueta, Federico García Lorca, Antonio Machado, Salvador, Dalí, Juan Negrín, Menéndez Pidal y tantos y tantos otros. En 1915 la Junta del Patronato abrió la Residencia de Señoritas dirigida por María Maeztu (1881-1948) con idéntica orientación que su semejante masculina.

Dependiente de la Junta para Ampliación de Estudios se crea el Instituto Escuela (1918), partiendo del interés de Cossío, con el objetivo de introducir en la enseñanza oficial los principios de la ILE (Jiménez, 1985). Se constituye un centro piloto para la transformación de la enseñanza básica (del parvulario hasta los 17 años) y se le confía a la ILE su dirección y puesta en marcha mediante José Castillejo. El Instituto tuvo una orientación de formación del profesorado de escuela secundaria, se exigían actividades de docencia y de investigación de forma paralela «aprender enseñando», se pagaban complementos y se requería dedicación exclusiva, facilitando visitas al extranjero. Esta experiencia supone la primera experiencia española en formación del profesorado en enseñanza media.

Rescatar a la ILE y llevarla a las actuales aulas universitarias del Grado de Magisterio significa adentrar a los futuros maestros y maestras en la génesis de su propia formación, ya que la ILE reabrió el debate sobre la importancia de formar buenos maestros y maestras, abrió la posibilidad de trabajar en las 
escuelas desde los métodos activos, coe-ducativos y experienciales, algo que se ha retomado actualmente en la formación inicial del profesorado, una formación encuadrada en el EEES.

\section{LA VOZ DE LA ILE, LA ORALIDAD HECHA CONOCIMIENTO}

La historia de vida es una estrategia narrativa (técnica clave en el modelo aquí presentado) y a partir de ella se proyectan valores humanos, sentimientos, contradicciones y los patrones significativos de una determinada cultura. Cuando hablamos desde las historias de vida, nos hacemos eco de datos que provienen de la vida cotidiana, del sentido común, de las explicaciones y reconstrucciones que el individuo hace sobre su tránsito de vida (Ruiz Olabuénaga, 2012). Teniendo en cuenta esto, accedimos a los informantes y protagonistas activos que formaron parte de la ILE, en este caso los alumnos y alumnas que estuvieron bajo el paradigma institucionalista tanto en el contexto español como en el argentino ${ }^{9}$. Nos aproximamos a las historias de vida de los informantes y nos ofrecieron un marco interpretativo a través del cual poder tener un mayor acceso a las prácticas pedagógicas reales de los institucionalistas. "En los relatos personales se dieron prioridad a las explicaciones individuales de las acciones didácticas" (Chárriez, 2012, 52). Desde este planteamiento acercamos ahora la ILE, sus principios y prácticas innovadoras en voz de Luis de Azcárate.

Luis de Azcárate es descendiente directo de uno de los fundadores de la ILE. Así nos lo hace saber al abordar su vida educativa:

Nosotros descendemos uno de los fundadores de la Institución, que fue Gumersindo de Azcárate, ese era tío abuelo mío. Era tío, del tío Pablo y tío Pablo le sustituyó un poco en toda la vida política. Este era un hombre liberal, que jugó un papel importante dentro del desarrollo de las tendencias liberales en el siglo XIX y que fundó junto con Don Francisco y con Salmerón, y con Castro y con Sanz del Río, pues fundaron la Institución Libre de Enseñanza. (...) Mi tío Pablo había sido Catedrático en la Universidad de Santiago, luego había optado por otra Cátedra y estuvo en Granada, y luego le ofrecieron el trabajo la Sociedad de Naciones, y se fue a la Sociedad de Naciones de Ginebra. (Entrevista, diciembre de 2014)

\footnotetext{
9 Tocamos el marco argentino ya que allí se exiliaron en tiempo Guerra Civil y Dictadura Franquista algunos integrantes de la ILE como fue el caso de Lorenzo Luzuriaga y $\mathbf{M}^{\mathrm{a}}$ Luisa Navarro.
} 
(...) Yo me eduqué en la Institución Libre de Enseñanza y yo termino en la Institución Libre de Enseñanza en julio. Soy la última generación que salió de la séptima clase, que sería la última. (Entrevista, diciembre de 2014)

Constituye una de las últimas voces vivas de esta Institución. En su narración subraya la importancia de las experiencias educativas activas en las cuales se desarrollaban los principios activos pedagógicos a partir de la metodología a desarrollar en la escuela republicana. En estas salidas se aprendía a partir de lo intuitivo, también a partir de la experiencia y la observación de obras de arte y mediante el contacto con la Naturaleza. La discusión y el debate eran otros principios pedagógicos sobre los que se enseñaba a pensar:

(...) Los domingos salíamos de la plaza Colón, del Instituto Escuela, a esquiar a la sierra, (...) pero la pedagogía de la Institución no era solo clase, sino también en el recreo (...) El jardín de la Institución era un centro de enseñanza directa.

Íbamos de excursión, algunas veces en la sierra teníamos una casa, pero íbamos a Toledo o a Ávila, al Escorial o a Aranjuez, Al Pardo, es decir, a lugares cerca. Hubo una vez una excursión fuera de lo normal a Guadalupe, pero claro el transporte era no fácil.

En la Institución no teníamos exámenes. En la Institución para enseñarnos era la explicación que nos daba el profesor del tema que fuese, historia o de matemáticas, los apuntes que nosotros tomábamos en el cuaderno y las preguntas que nos hacía el profesor o las preguntas que hacíamos nosotros al profesor, a través de las cuales se establecía una cierta discusión.

A nosotros el señor Giner que era nuestro profesor de arte, pues nos daba las clases en el museo de reproducciones, que era el museo donde había las reproducciones de las esculturas clásicas griegas, latinas de todo, que estaba en aquel momento, lo que estaba en el Retiro, donde estuvo la obra de Guernica.

Pero nos explicaban el Museo del Prado, entrábamos por donde estaba la estatua Velázquez, y nos llevaban a todas las salas.

En Toledo, el Toledo de antes de la guerra. Donde está, os lo dije en la Posada de la Sangre. Enfrente de la Posada de la Sangre, o el convento o el manicomio, ahí estaban todos los cuadros del Greco. Nos explicaba el Greco a tope y eso lo hacía el señor Cossío. (Entrevista, diciembre de 2014)

Y con estas salidas se educaba en el respeto a la Naturaleza, pilar pedagógico central en los preceptos educativos para la enseñanza. Además la Naturaleza había que respetarla, era un bien común a cuidar: 
En el jardín, había un muro de ladrillos y ahí una yedra preciosa verde que lo trepaba, pero una yedra que toda la parte de abajo del muro estaba totalmente cubierta de la yedra ¿no? Entonces, claro jugábamos al fútbol o jugábamos con una pelota. Y de repente la pelota llegaba a la yedra. Entonces uno de los clásicos de la Institución, el señor Rego, profesor, recuerdo todavía la voz del señor Rego decir "iLa yedra!". Ya se fastidió la pelota. Nosotros no podíamos ir a sacar la pelota de la yedra, porque la yedra había que respetarla y si sacábamos la pelota y una hojita de la yedra caía, pues había bronca y media. Porque había que respetar la naturaleza.

Cuando ibas de excursión, simplemente a la Casa de Campo o al Pardo, lo que no podías era dejar papeles porque el campo no se podía ensuciar. El campo había que respetarlo. Los papeles había que recogerlos rigurosamente, donde tenías envuelta la tortilla, tenía que quedar todo totalmente igual o más limpio que cuando te habías sentado a comer. Te enseñaban a respetar el campo, a los árboles, ¿quitar una rama de un árbol? No, nada de eso. O el tomillo y todo eso, pues había que dejarlo ahí, tan tranquilo. Porque si llevabas un poco de tomillo a tu madre pues era agradable, pero no, no. Te enseñaban a contemplar una puesta de sol... La Naturaleza era una parte muy importante de la pedagogía de la ILE. (Entrevista, diciembre de 2014)

Igualmente importante era el respeto hacia "el otro". El aprendizaje por las normas y los valores medioambientales se han reflejado anteriormente, pero esos valores medioambientales no eran los únicos que se aprendían, había otros que se extendían a lo personal. Estos valores estaban unidos al principio de igualdad:

Cuando había un conflicto, no, era "perdona", la mano y ya está, había un respeto, te educaban a respetar. (...) pues teníamos que llegar a un acuerdo. Te enseñaban a respetar la opinión del otro". "Cuando eran las diez y media u once pues había un recreo grande, pues tenías cierta hambre. Allí nadie se quedaba sin un pedazo de pan porque a alguien se le había olvidado el bocadillo... Así que se le decía "toma". (Entrevista, diciembre de 2014)

Porque una de las cosas que nos enseñaban en la Institución era a no mentir. Cuando hacíamos una barrabasada pues, decían "¿quién ha hecho esto?" - "He sido yo. Yo lo he hecho". No mentíamos ni acusábamos. El acusica estaba muy mal visto en la Institución. (Entrevista, diciembre de 2014)

Luis de Azcárate, a través de la entrevista, también nos deja hallazgos históricos relacionados con las Ciencias, y sería difícil saber de ellos sino fuera por el ejercicio de memoria realizado por él en el relato de su vida académica: 
En la subida a Navacerrada, en la carretera que va desde Madrid a Navacerrada, como dos o tres vueltas antes de llegar al puerto hay una fuente en el lado izquierdo de granito, muy sencilla, con un agua rica, riquísima, fría. Esa fuente era la fuente de los geólogos, la vi yo hacer. Era una fuente donde en el lado derecho, si os fijáis, ahí hay nombres, pero ya no se ven, grabados en la piedra de granito. Ahí hay nombre de la Institución, de la gente de la Institución, el señor Linares, eran los geólogos. Pero esos son los primeros, naturalistas se llamaban también. Eran los primeros ecólogos o ecologistas de la ciencia española. (Entrevista, diciembre de 2014)

El Museo de Ciencias Naturales lo hizo el señor Bolívar, que fue exiliado después a México, con el diplodocus aquel monstruoso... El señor Sols fue profesor, yo creo, del Instituto de Toledo. Era todo un desarrollo nuevo de la enseñanza de los animales, de los bichos, de los polipitos. (...) había un laboratorio que sigue el edificio Macpherson de historia natural, en donde estaban conservados cantidad de animalitos que nosotros les decíamos polipitos. (Entrevista, diciembre de 2014)

Azcárate nos deja una bonita y precisa reflexión sobre lo que a él le aportó su presencia en la ILE. Conecta con el presente haciendo un puente temporal que nos debe hacer reflexionar sobre la importancia de la educación en la actualidad:

Mira una cosa que es importante, para aclarar lo que era la Institución. La Institución era un lugar donde no se hacía política. (...) A mí, me aportó la ILE el ser persona. El ser persona y el entender que había que dedicar la vida a cambiar la situación económica y política de España. (...) La falta de respeto a la memoria de nuestros maestros, lo que ha sido la educación... hay que recupéralo. (...) Yo creo que la educación en gran parte puede cambiar el país. (Entrevista, diciembre de 2014)

\section{CONCLUSIONES}

Solo hemos aproximado una pequeña parte del gran relato de vida que Luis de Azcárate nos ha ofrecido en su propia casa durante una fría mañana de diciembre en 2014. De sus palabras se deriva añoranza por una época pasada poco estudiada en la historia educativa, pero importante para el presente educativo. En el análisis de los fragmentos de la entrevista podemos identificar principios pedagógicos en las actuales leyes educativas (LOE LOMCE). También, contenidos en forma de valores y normas que se rescataron en la LOGSE, por ejemplo el cuidado de la Naturaleza como un bien patrimonial. Actualmente aparecen corrientes y modelos para educar a 
niños y niñas que tienen su origen en la ILE. Nos referimos a la pedagogía museística (Huerta, 2011), el patrimonio integrado (Melgar \& Donoso, 2011), o los movimientos de educación para el desarrollo sostenible (Pinzolas; Conde; Artigas \& Olmo, 2011). Volver la mirada pedagógica al pasado, implica recuperar memoria histórica y escolar, también las voces que permanecen al margen del sistema formal de enseñanza pero que forman parte del su imaginario y lo hicieron posible. Trabajar ofreciendo voces reales con experiencia educativa transforma y hace aportes pedagógicos que no se podrían hacer de otra manera. Enriquecen los conocimientos que surgen a través de las instituciones sociales y educativas.

Aplicar el modelo de memoria educativa y pedagógica en la formación inicial de maestros y maestras aporta, además, perspectiva y dimensión interdisciplinar a los hechos educativos y a las figuras que originaron estos hechos en forma de métodos para la enseñanza, tendencias educativas o materiales didácticos. Esa interdisciplinariedad atraviesa lo etnográfico, lo histórico, lo pedagógico; implica que el alumnado acceda a fuentes de transmisión de datos hoy todavía vivas y que aprecien bienes culturales surgidos en otros siglos. Aporta la compresión de fenómenos educativos (como el de la ILE) que fueron originados por la confluencia de múltiples fenómenos (culturales, sociales, económicos...) y sobre todo les ofrece el acceso a un patrimonio conformado por hitos, personajes y hallazgos difíciles de identificar sino es a partir de la puesta en marcha de este modelo.

\section{REFERENCIAS BIBLIOGRÁFICAS}

Aguilar, M. A. (2002). Fragmentos de la memoria colectiva. Athenea Digital. $2,1-11$.

Aguirre, M. E. (Coord.), (2013). Lecturas in-apropiadas desde la historia, la educación y la cultura. México D.F.: Ediciones Díaz de Santos y Universidad Nacional Autónoma de México.

Bejarano, M. y Lirio, J. (2008). La utilización de problemas auténticos en la Enseñanza Superior. En Escribano, A. y Del Valle, A. El aprendizaje basado en problemas. Una propuesta metodológica en Educación Superior (pp. 35-52). Madrid: Narcea.

Bejarano, M., Mateos, A. y Rodrígues, A. (2015). Los trabajos fin de grado en la formación inicial de los y las docentes. Hacia un modelo basado en la competencia investigadora. En Rodríguez Torres J. (Coord.). Experiencias en la adaptación al EEES (pp. 121-133). Madrid: Mac Graw Hill. 
Benadiba, L. y Plotinsky, D. (2001). Historia Oral. Construcción del archivo histórico escolar. Una herramienta para la enseñanza de las Ciencias Sociales. Buenos Aires-México: Novedades Educativas.

Bolívar, A. (2008). El discurso de las competencias en España: Educación Básica y Educación Superior. Revista de Docencia Universitaria, 6, (2), $1-23$.

Bolívar, A. y Domingo, J. (2006). La investigación biográfica y narrativa en Iberoamérica: Campos de desarrollo y estado actual. Forum: Qualitative Social Research, 7, (4), 1-43.

Bustos, G. (2010). La irrupción del testimonio en América Latina: intersecciones entre historia y memoria. Presentación del dossier "Memoria, historia y testimonio en América Latina". Historia Crítica, $40,10-19$.

Cabero, J. y Gisbert, M. (2005). Formación en Internet. Guía para el diseño de materiales didácticos. Sevilla: MAD.

Cossío, M. B. (1966). De su jornada. Madrid: Aguilar.

Chárriez, M (2012). Historias de vida. Una metodología de investigación cualitativa. Revista Griot, 5, (1), 50-67.

Dávila, P. V. (2011). La documentación narrativa de experiencias pedagógicas. Una estrategia de reposicionamiento de saberes, conocimientos y actores en el campo de la formación docente. Revista Educación y Pedagogía, 23 (61), 145-155.

Diamant, A. (2010). Testimonios de enseñar y aprender. Buenos Aires: Teseo.

Diamant, A. (2014). La Historia Oral. Buenos Aires: Biblioteca Nacional de Maestros. Gobierno de la Nación.

Escribano, A. y Del Valle, A. (2008). El aprendizaje basado en problemas. Una propuesta metodológica en Educación Superior. Madrid: Narcea.

Fernández Enguita, M., Mena, L. y Riviere, J. (2010). Fracaso y abandono escolar en España. Barcelona: Fundación la Caixa.

Grangeiro, D. (2011). Documentación narrativa de experiencias pedagógicas de docentes jubilados. Revista de Educción y Pedagogía, 23 (61), 123132.

Gómez, J., Ramírez, R., Rodríguez, A. y Romero, J. G. (2004). Manual básico de Aprendizaje Basado en Problemas. México: UNAM.

Gómez, E. (2012). Oralidad y memoria: sobre los testimonios verbales del pasado. ETNICEX, 4, 19-39.

Huerta, R. (2011). Maestros, museos y artes visuales. Construyendo un imaginario educativo. Arte, Individuo y Sociedad, 23 (1), 55-72.

Jiménez-Landi, A. (1973). La Institución Libre de Enseñanza. Madrid: Taurus.

Jiménez-Landi, A. (2010). Breve historia de la Institución Libre de Enseñanza (1896-1939). Madrid: Tébar. 
Jiménez, A. (1985). El Krausismo y la Institución Libre de Enseñanza. Madrid: Cincel.

Latorre, A. y González, R. (1992). El maestro investigador: La investigación en el aula. Barcelona: Graó.

Luzuriaga, L. (2002). La escuela nueva pública. Madrid: Losada

Melgar, M. F. y Donoso, D. S. (2011). Salir del aula... Aprender de otros contextos: Patrimonio natural, museos e internet. Revista Eureka sobre Enseñanza y Divulgación de las Ciencias, 8 (3), 323-333.

Meyer, E. (1991). La historia oral en Latinoamérica y el Caribe. Historia y Fuente Oral, 5, 139-144.

Meyer, E. (1995). Los nuevos caminos de la historia oral en América Latina. Historia y Fuente Oral, 13, 97-103.

Millán, F. (1983). La Revolución Laica de la Institución Libre de Enseñanza. Valencia: Fernando Torres.

Otero, E. (2007). Las primeras expediciones de maestros de la Junta para la Ampliación de Estudios y sus antecedentes: los viajes de estudio de Cossío entre 1880 y 1889. Revista de Educación, Número extraordinario, 45-66.

Palacios, L. (1979). José Castillejo. Última etapa de la Institución libre de Enseñanza. Madrid: Narcea.

Pinzolas, J. A., Conde, O., Artigas, T. y Olmo, D. (2011). Los huertos escolares, semillas de cambio en las aulas. Fertilidad de la tierra: revista de agricultura ecológica, 44, 54-56.

Portal, E. (2011). Metodología colaborativa: el compromiso y el consenso de los grupos de aprendizaje. En Morales S. (Coord.), Nuevos contextos de enseñanza y aprendizaje (pp. .139-153). Buenos Aires: Miño y Dávila.

Pujadas, J. (2000). El método biográfico y los géneros de las memorias. Revista de Antropología Social, 9, 127-158.

Riesco, M. (2008). El enfoque por competencias en el EEES y sus implicaciones en la enseñanza y el aprendizaje. Tendencias Pedagógicas, 13, 79-105.

Rodríguez, J. (2014). Experiencias en la adaptación al EEES. Madrid: McGraw Hill.

Ruiz Olabuénaga, J. I. (2012). Metodología de la Investigación Cualitativa. Bilbao: Universidad de Deusto.

Sánchez de Madariaga, E. (2012). Las Maestras de la República. Madrid: Catarata.

Sánchez Morillas C. M. (2010). La figura de la maestra rural en la Segunda República. Revista de Antropología Experimental, 10, 119-128. 
Sánchez Sánchez, I. (2012). Educación, Ciencia y Cultura en España: Auge y colapso (1907-1940). Ciudad Real: Centros de Estudios de Castilla-La Mancha.

Suárez, D. H. (2011). Indagación Pedagógica del mundo escolar y formación docente. La documentación narrativa de experiencias pedagógicas como estrategia de investigación formación-acción. Revista del Instituto de Investigaciones en Ciencias de la Educación, 30, 17-32.

Stenhouse, L. (1987). La investigación como base para la enseñanza. Madrid: Morata.

Walter, M. (2002). El potencial epistemológico de la historia oral: algunas contribuciones de Silvia Rivera Cusicanqui. En Mato, D. (Coord.). Estudios y Otras Prácticas Intelectuales Latinoamericanas en Cultura y Poder. Caracas: Consejo Latinoamericano de Ciencias Sociales (CLACSO) y CEAP, FACES, Universidad Central de Venezuela. 